\title{
Balanced Permutation
}

National Cancer Institute

\section{Source}

National Cancer Institute. Balanced Permutation. NCI Thesaurus. Code C64218.

A method for reducing bias in which half of the treatment arrays swap with half of the control arrays in each permutation. 